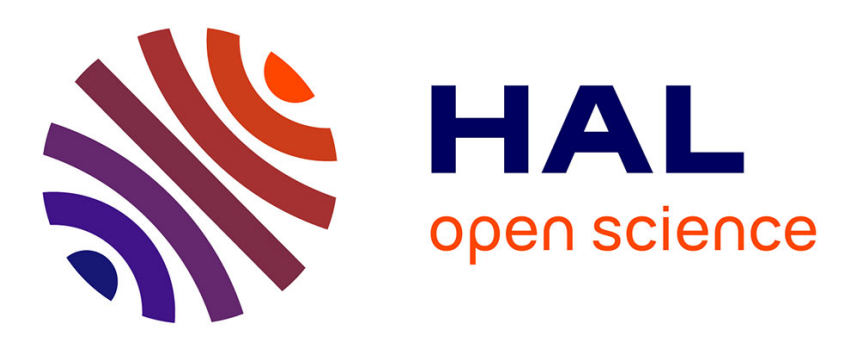

\title{
Analyse de la réception des messages médiatiques Récits rétrospectifs et verbalisations concomitantes \\ Didier Courbet, Marie-Pierre Fourquet-Courbet
}

\section{To cite this version:}

Didier Courbet, Marie-Pierre Fourquet-Courbet. Analyse de la réception des messages médiatiques Récits rétrospectifs et verbalisations concomitantes. Communication \& langages, 2009, 161, pp.117135. sic_00459181

\section{HAL Id: sic_00459181 \\ https://archivesic.ccsd.cnrs.fr/sic_00459181}

Submitted on 23 Feb 2010

HAL is a multi-disciplinary open access archive for the deposit and dissemination of scientific research documents, whether they are published or not. The documents may come from teaching and research institutions in France or abroad, or from public or private research centers.
L'archive ouverte pluridisciplinaire HAL, est destinée au dépôt et à la diffusion de documents scientifiques de niveau recherche, publiés ou non, émanant des établissements d'enseignement et de recherche français ou étrangers, des laboratoires publics ou privés. 
Référence à citer pour ce texte : FOURQUET-COURBET, M.P. et COURBET, D. (2009), Analyse de la réception des messages médiatiques, Récits rétrospectifs et verbalisations concomitantes, Communication et Langages ${ }^{1}$, issue 161, septembre 2009, pp. 117-135.

\title{
Analyse de la réception des messages médiatiques
}

Récits rétrospectifs et verbalisations concomitantes

\author{
Marie-Pierre Fourquet-Courbet \\ Université d'Avignon et des pays du Vaucluse \\ Laboratoire Culture et communication
}

Didier Courbet

Aix-Marseille Université

IRSIC-Médi@sic

\section{Résumé}

On connait mal les processus cognitifs et affectifs mis en œuvre par les sujets sociaux lors du contact avec les dispositifs médiatiques, notamment parce que les méthodes pour étudier la dynamique des processus de réception individuelle sont insuffisamment développées. L'article propose de contribuer à combler cette carence méthodologique en présentant deux méthodes qui permettent d'étudier et «pister» les processus : la méthode des Récits de Réception Rétrospectifs (RRR) et la méthode d'Etude des Cognitions verbalisées concomitantes En Réception (ECER). En nous appuyant sur deux études de cas, nous expliquons leurs fondements théoriques et épistémologiques ainsi que les modalités pratiques de recueil et d'analyse. Par une analyse comparative, nous précisons ensuite les conditions qui garantissent la validité scientifique et la bonne application des deux méthodes pour étudier la réception de la communication médiatique.

Les chercheurs en sciences de communication s'accordent sur le fait qu'une étude communicationnelle portant sur la globalité d'un phénomène médiatique s'intéresse à trois pôles, la production, le contenu médiatique lui-même et sa réception. Force est de constater que la réception demeure le pôle le moins investi des trois par la recherche française. La réception est cependant fondamentale. Les théories de la communication médiatique expliquent que la signification sociale d'un «message» médiatique naît de l'interaction entre un texte, plus généralement un dispositif médiatique, et des sujets sociaux ou des 
publics «récepteurs», socialement contextualisés. Ces publics sont considérés comme co-producteurs de la signification ${ }^{1}$. De plus, il ne fait plus de doute aujourd'hui qu'un grand nombre de contenus médiatiques (images violentes, publicité, actualités, idéologies sous-jacentes aux émissions...) forment, renforcent ou modifient les représentations, les idéologies et les actions sociales ${ }^{2}$.

Dans les recherches sur la réception en sciences de la communication, différentes ressources théoriques sont utilisées pour étudier de manière complémentaire les phénomènes de réception médiatique. Pour étudier les «effets macroscopiques » à moyen et long termes des médias sur la société, les ressources théoriques sont plutôt sociologiques. Par exemple, les recherches s'insérant dans le contexte théorique de la spirale du silence ${ }^{3}$ montrent que les médias de masse agissent sur l'espace public en le vidant de certaines opinions, en modifiant lentement les représentations et l'idéologie sociales. Dans une perspective différente, l'ethnographie de la réception ${ }^{4}$ montre que les contenus médiatiques sont interprétés différemment selon les groupes ou les diasporas qui co-construisent de la signification sociale après les phases de contact individuel avec le média. Les approches sociologiques et ethnographiques ne théorisent qu'une partie du processus de réception, à partir du moment où les sujets sociaux s'éloignent du média, forment des discours ou engagent des conversations à propos de ce qu'ils ont $\mathrm{vu}^{5}$. Dans ces deux approches, les processus qui opèrent en amont, au moment où les sujets sociaux sont en contact avec le média sont considérés comme « des boîtes noires ».

Pour supprimer ce point aveugle, les sciences de la communication s'appuient sur la psychologie sociale et de la psychologie pour théoriser les processus intra- et inter-individuels et les processus socio-cognitifs et socio-affectifs, mis en oeuvre par les sujets sociaux au moment même ou immédiatement après le contact avec les médias ${ }^{6}$. Comment les sujets sociaux reçoivent-ils les messages médiatiques ? Par quels mécanismes construisent-ils du sens lors du contact avec les médias ? Ces questions sont d'autant plus importantes que la réception des sujets sociaux précède et détermine en partie le processus de co-construction sociale de la signification et les effets à moyen et long termes au sein des micro- et macro-groupes sociaux. Avant toute circulation, interprétations, effets sociaux et culturels, les messages et les textes médiatiques sont reçus par des individus sociaux, parmi lesquels des leaders d'opinion, qui peuvent ensuite faire entrer et relayer l'information de provenance médiatique dans les réseaux et les groupes.

Cependant, les théories de la réception des sujets sociaux restent insuffisamment développées. Une des

\footnotetext{
${ }^{1}$ S. HALL, “Encoding/Decoding TV discourse ”, CCCS, University of Birmingham, 1973.

${ }^{2}$ P. MARCHAND, P. (Dir.), Psychologie sociale des médias, Presses Universitaires de Rennes, 2004. D. COURBET, M.P. FOURQUET-COURBET (Dir.) La Télévision et ses influences, Bruxelles, De Boeck Université/INA, 2003 ;

3 E. NOELlE-NEUMANN, E., “ The Spiral of Silence: A Theory of Public Opinion ”, Journal of Communication, vol.24, pp. 43-51, 1974.

${ }^{4}$ D. DAYAN, “ Sociologie des médias : le détour par l'ethnographie”, pp. 445-465, in: Moscovici S. Buschini F., eds, Les méthodes des sciences humaines, Paris, Presses universitaires de France, 2003.

${ }^{5}$ D. BOULLIER, La télévision telle qu'on la parle. Trois études ethnographiques, Paris, Éd. L'Harmattan, 2003

${ }^{6}$ COURBET, D., M.-P. FOURQUET-COURBET, C. CHABROL, « Sujets sociaux et médias : débats et nouvelles perspectives en sciences de l'information et de la communication », Questions de Communication, 10, pp. 157-179, 2006.
} 
cause est une carence méthodologique : les chercheurs ne disposent pas de méthode pertinente, suffisamment fondée épistémologiquement et théoriquement pour étudier les processus cognitifs et affectifs intra- et inter-individuels se déroulant lors du contact avec les médias. L'objectif de l'article est de contribuer à combler cette lacune en présentant deux méthodes d'études de la réception permettant de pister les processus de réception individuelle lors du contact avec les messages : la méthode des Récits de Réception Rétrospectifs (RRR) et la méthode d'Etude des Cognitions verbalisées concomitantes En Réception (ECER).

Nous présentons chaque méthode afin que le lecteur puisse l'utiliser de manière réfléchie et réflexive. Nous explicitons leurs fondements, leurs apports potentiels mais également leurs limites pour étudier la réception de la communication médiatique. On ne peut évaluer la qualité d'une méthode qu'au regard des objectifs théoriques du chercheur. Chaque méthode est illustrée par une recherche empirique concernant un cas de réception médiatique. Dans la première partie, nous présentons la méthode des RRR puis, dans la deuxième partie, la méthode ECER. Nous expliquons systématiquement, pour les deux méthodes, leurs principes, leurs fondements et présupposés épistémologiques et théoriques, les procédures de passation, de recueil et d'analyse des informations. Dans la troisième partie, nous comparons les deux méthodes quant à leur intérêt épistémologique et théorique, d'une part et, d'autre part quant aux conditions nécessaires pour garantir leur validité et leur bonne application dans le cadre des études de la réception de la communication médiatique.

\section{La méthode des récits de réception rétrospectifs (RRR)}

La méthode d'étude des récits de réception rétrospectifs (RRR) est une méthode de recueil et d'analyse de récits de personnes rapportant rétrospectivement leur vécu expérientiel, leurs réactions, leurs représentations et leurs comportements individuels et sociaux lors de la réception d'un événement médiatique unique et important (e.g. les attentats de New York en 2001, la mort de la princesse Diana, l'élection d'un Président de la République). A partir d'un échantillon diversifié de sujets sociaux contextualisés, elle permet la compréhension des phénomènes psychologiques et sociaux déclenchés ou en lien avec des événements médiatiques majeurs.

\section{Principes et fondements}

Doublement inspirée par la méthode anthropologique des récits de $\mathrm{vie}^{7}$ et par la méthode des protocoles verbaux rétrospectifs utilisés en ergonomie cognitive ${ }^{8}$, nous avons ajouté à la méthode des RRR des éléments théoriques issus des psychologies cognitive, sociale et des émotions. Les

\footnotetext{
7 J. POIRIER, S. CLAPIER-VALADON, P. RAYBAUT, Les récits de vie. Théorie et pratique, Paris, Presses universitaires de France, 1983.

${ }^{8}$ H. KUUSELA, P. PAUL, «A comparison of concurrent and retrospective verbal protocol analysis », The American Journal of Psychology, Vol. 113 º3, p.387-404, 2000.
} 
événements socio-médiatiques majeurs, s'ils sont surprenants, importants et fortement émotionnants sont inscrits dans la mémoire autobiographique des individus sous forme de souvenir flash (flashbubl memory). Ce type de souvenirs est très détaillé, imagé et conserve les informations contextuelles sur l'événement «presque comme la réalité elle-même »". Les recherches montrant la bonne fiabilité de la mémoire flash permettent d'accorder une validité théorique solide aux RRR. Les discours autoreportés que les sujets donnent à partir de leur souvenir flash sont fidèles à la manière dont l'événement s'est effectivement déroulé dans le passé, y compris après un laps de temps élevé ${ }^{10}$. Dans une logique proche de celle de la cognition située ${ }^{11}$ et selon une perspective d'épistémologie herméneutique, le chercheur essaye, par un principe d'empathie, de comprendre la complexité du phénomène de réception via les RRR. Comment et par quels processus socio-cognitifs et socioémotionnels individuels et sociaux, les sujets récepteurs ont-ils construit la signification de l'événement médiatique ? Comment ont-ils réagi et comment ont-ils vécu la réception en direct, au sein de leur contexte de vie particulier, en fonction de leur personnalité, de leur histoire personnelle, des interactions sociales qu'ils ont eues avec leur entourage?

\section{La réception des attentats de New York en 2001}

Nous illustrons la méthode des RRR par une enquête exploratoire que nous avons menée sur la réception télévisuelle des attentats de New York en $2001^{12}$. L'objectif de cette recherche empirique est d'expliquer les réactions de téléspectateurs français face aux images montrant en direct les attentats de New York. Il s'agit d'étudier leurs réception par des personnes qui ont appris l'événement par la télévision, en regardant les images de l'avion percutant une des tours en direct, puis au cours des 10 minutes qui ont suivi. Les RRR sont recueillis deux mois après l'événement, en novembre 2001. Ce délai incompressible pour la préparation de l'enquête est convenable au regard de la bonne stabilité des souvenirs flash.

\section{Procédure pratique de passation et matériel.}

Afin de favoriser le rappel des souvenirs ${ }^{13}$, l'enquête a lieu dans le même contexte socio-physique que la réception initiale, au domicile des sujets. L'intervieweur apporte un enregistrement des images de l'événement qui seront passées sur la même télévision que celle ayant été utilisée lors de la réception initiale. On procède à un enregistrement sonore de l'entretien dont la durée varie, selon les personnes,

\footnotetext{
9 D.B. WRIGHT, G.D. GASKELL, «Flashbulb memories: Conceptual and methodological issues », Memory, 3, p. 67-80, 1995.

${ }^{10}$ F.J. BROWN, J. KULIK, « Flashbulb memory », Cognition, 5, p. 73-99, 1977

${ }^{11}$ L. SUCHMAN, Human-Machine Reconfigurations: Plans and situated actions, Cambridge University Press, New York, 2007.

${ }^{12}$ D. COURBET, M.P. FOURQUET, « Réception des images d'une catastrophe en direct à la télévision. Etude qualitative des réactions provoquées par les attentats du 11 septembre 2001 aux Etats-Unis au travers du rappel de téléspectateurs français », Revue Européenne de Psychologie Appliquée, p.21-41, 2003.

${ }^{13}$ E. TULVING, D.M. THOMSON, «Encoding specificity and retrieval processes in episodic memory », Psychological Review, 80, p. 352-373, 1973.
} 
de 30 mn à 3 heures.

\section{Procédure de recueil des informations et rôle de l'enquêteur}

Après avoir mis la personne en confiance, l'intervieweur lui demande de parler librement, sans se censurer, en essayant de se rappeler le plus fidèlement possible ses souvenirs. Afin de faciliter la récupération en mémoire autobiographique ${ }^{14}$, on demande d'abord à la personne de parler de ce qu'elle faisait avant de voir les images pour la première fois. Ensuite, on la ré-expose au contenu médiatique. A l'aide d'une procédure très peu dirigée, l'intervieweur demande à la personne de narrer ses réactions et comportements. L'intervieweur aide la personne à expliciter au mieux ses réactions en utilisant différents types de techniques verbales, para-verbales et non-verbales destinées à faciliter la production du récit : pratique de l'écoute active, reformulations, relances...

\section{Echantillonnage : taille et mode de sélection}

Après un tirage au sort dans l'annuaire téléphonique puis un premier filtrage téléphonique permettant d'éliminer les personnes n'ayant pas appris l'événement en voyant les images en direct à la télévision, 56 sujets ont été interrogés. L'échantillon est diversifié quant aux variables sexe, âge, PCS et type d'habitat.

\section{Une méthode d'analyse du discours spécifique}

Après retranscription, le chercheur étudie d'abord chaque discours dans sa singularité. Il effectue ensuite des analyses transversales, pour rechercher une macro-structure invariante sous-jacente à l'ensemble des données linguistiques recueillies. Dans l'étude de la macrostructure, il s'agit de repérer les différents processus de réception. Un processus est constitué de différentes phases qui se suivent chronologiquement et qui ont une continuité logique et homogène. Il s'agit, avant tout, de procéder à une analyse inter-processuelle portant sur l'articulation des processus entre eux et sur la logique qui sous-tend l'articulation. L'analyse s'effectue sans délinéarisation, en maintenant la chronologie des événements psychologiques et psychosociaux. On procède ensuite à une analyse intra-processuelle où on repère les noyaux de réactions (NR). Le NR est un ensemble de réactions psychologiques d'un individu pris dans un phénomène de réception télévisuelle. Le NR est souvent relié à un prédicat (e.g. un attribut) ou parfois à une proposition qui le caractérise. Le NR est constitué, d'une part, de traitements d'informations effectués en mémoire de travail et, d'autre part, des représentations qui résultent de ces traitements. Un NR individuel est soit cognitif (référent, informations associées aux représentations), soit affectif ou émotionnel, soit comportemental, constitué de conduites objectivables. Le NR “ social ” est lié à une communication avec l'entourage social.

On analyse enfin les phases, constituées de plusieurs NR qui, soit se suivent chronologiquement, soit

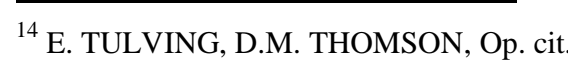


ont une homogénéité théorique entre eux. Dans ce dernier cas, au sein d'une même phase, on casse la linéarité et la suite chronologique du discours pour construire des NR. L'enchaînement discursif est donc souvent déstructuré.

\section{Trois processus constants}

Les résultats montrent que les personnes interrogées ont vécu, avec une chronologie identique, les mêmes trois grands processus (voir tableau 1). Afin de réduire la trop grande subjectivité dans les analyses de contenu, deux codeurs ont analysé les discours. Le coefficient de fidélité intercodeurs (calculé selon la formule : nombre d'accords sur les classifications entre les deux codeurs / nombre total de classifications dans l'analyse) est de 0.91 . Comme le seuil d'acceptabilité est à $0.80^{15}$, on a une bonne homogénéité des analyses effectuées par les deux codeurs.

Tableau 1. Extrait des résultats de l'analyse de contenu

Premier processus mis en place pendant la première minute :

primat des émotions négatives (deux phases)

Phase 1. Réactions individuelles et préoccupations autocentrées

\begin{tabular}{|l|l|}
\hline $\mathbf{N R}^{*}$ & $\mathbf{F}^{*}$ \\
\hline Fortes réactions émotionnelles négatives & 40 \\
\hline Première construction de représentations cognitives de la tragédie & 30 \\
\hline
\end{tabular}

Phase 2 : Prise en compte de l'environnement social

\begin{tabular}{|l|l|}
\hline $\mathbf{N R}^{*}$ & $\mathbf{F}^{*}$ \\
\hline Sentiment négatif lié à l'impossibilité d'aider les victimes & 27.5 \\
\cline { 2 - 2 } Brutales réactions de peur causées par présence d'une connaissance à N.Y. & 2.5 \\
\hline Calculs marginaux & $\mathbf{1 0 0}$ \\
\hline
\end{tabular}

Calculs marginaux

Deuxième processus mis en place à partir des deux-trois minutes suivantes :

Interprétations, interactions socio-émotionnelles et socio-cognitives (trois phases)

Phase 1: Seconde tentative d'interprétation individuelle

\begin{tabular}{|l|l|}
\hline $\mathbf{N R}^{*}$ & $\mathbf{F}^{*}$ \\
\hline Seconde construction de représentations cognitives individuelles & 28 \\
\hline Etat très provisoire et insatisfaisant des représentations peu élaborées de l'événement & 3 \\
\hline
\end{tabular}

Etat très provisoire et insatisfaisant des représentations peu élaborées de l'événement

Phase 2 : Recherche d'interactions sociales et d'échanges socio-émotionnels

\begin{tabular}{|l|l|}
\hline $\mathbf{N R}^{*}$ & $\mathbf{F}^{*}$ \\
\hline Besoin d'échanges socio-émotionnels et recherche de communication & 12 \\
\hline Satisfaction à apprendre la catastrophe à un de ses proches & 3 \\
\hline Extériorisation et partage social des émotions & 12.5 \\
\hline Comparaison sociale et identité personnelle & 2.5 \\
\hline
\end{tabular}

\begin{tabular}{|l|l|}
\hline Comparaison sociale et identité personnelle & 2.5 \\
\hline
\end{tabular}

Phase 3 : Recherche d'une co-construction des représentations cognitives par interactions sociales

\begin{tabular}{|l|l|}
\hline NR* & F* $^{*}$ \\
\hline Plus grande prise de conscience de la réalité de la tragédie (besoin d'ancrage dans le réel) & 6 \\
\hline Normalisation de ses propres représentations en fonction de celles des autres & 20 \\
\hline Réévaluation sociale du danger & 8 \\
\hline Mise en forme mémorisable de l'événement & 5 \\
\hline Calculs marginaux & $\mathbf{1 0 0}$ \\
\hline
\end{tabular}

15 C.P. SMITH, « Content Analysis and Narrative Analysis », in H.T. REIS, C. JUDD (Eds.), Handbook of research methods in social and personality psychology, NY Cambridge University Press, p. 313-335, 2000. 
Troisième processus mis en place après sept-huit minutes :

Réévaluation de la dangerosité à moyen terme et première peur (noyaux de réactions affectives et cognitives)

\begin{tabular}{|l|l|}
\hline NR* & $F^{*}$ \\
\hline Plus grand apaisement émotionnel & 9 \\
\hline Empathie et tristesse pour les Américains concernés & 22 \\
\hline Apparition d'une légère émotion de peur & 4 \\
\hline Ambivalence affective & 0.5 \\
\hline Traitement d'un plus grand nombre d'informations et plus grande capacité à les traiter & 11 \\
\hline Enrichissement des représentations cognitives de l'attentat & 24 \\
\hline Accroissement de la menace perçue à moyen terme & 11.5 \\
\hline Forte dépendance médiatique de l'individu & 18 \\
\hline Calculs marginaux & $\mathbf{1 0 0}$ \\
\hline * Notes : NR signifie noyau de réactions ; F : Fréquence d'occurrence du NR par rapport \\
aux NR totaux du processus (en pourcentage).
\end{tabular}

\section{Emotion et partage}

Dans le premier processus, lors de la première minute, l'individu est soudainement dans un état de fort éveil, de profonde stupéfaction, dont la valence affective est fortement négative, mais pas dans un état de peur. Les personnes trouvent l'événement complètement irréel : beaucoup utilisent la métaphore du cinéma pour exprimer cette irréalité. Les téléspectateurs tentent ensuite de donner une première interprétation de l'événement avec des questions peu élaborées : combien de morts ? Est-ce un attentat? Un phénomène d'empathie vis-à-vis des personnes prisonnières des tours débute à ce moment. Des sensations plutôt douloureuses naissent de sentiments d'impuissance et de résignation face à l'événement.

Lors du deuxième processus, deux ou trois minutes après le choc, les téléspectateurs commencent à développer les premières véritables réflexions cognitives élaborées mais leur construction individuelle de signification est insatisfaisante : la plupart des personnes interrogées ressentent alors un vif besoin soit de parler avec les personnes présentes, soit de téléphoner. Certains mentionnent le plaisir qu'ils ont eu à apprendre l'événement aux autres. Dans ce cas, c'est le fait de voir les vives réactions émotionnelles qu'ils génèrent qui les intéresse particulièrement : la personne qu'ils préviennent citera leur nom lorsque, plus tard, elle expliquera la manière dont elle a appris l'événement. Ainsi la communication interpersonnelle a-t-elle deux effets immédiats : a) un effet de référence à soi où l'individu dans un élan autocentré, voire narcissique, tente immédiatement d'avoir un rôle dans ce «phénomène planétaire »; b) un effet où le partage social crée une mémoire partagée, renforce la micro-culture commune et la cohésion entre ces deux personnes.

\section{La construction d'un récit, dans l'interaction sociale}

Sur le plan individuel, la verbalisation, l'extériorisation et le partage social des émotions négatives permettent d'en réduire l'intensité. La communication interpersonnelle au cours de la réception aurait une triple fonction identitaire en liant les expressions sociales des émotions et le concept de soi. Premièrement, les échanges sociaux permettent à chaque individu, par auto-attribution, de situer ses propres réactions par rapport à celles des autres. Deuxièmement, chez certaines personnes, il y a une recherche de légitimation sociale des émotions ressenties individuellement (ont-ils " raison " ou " tort " de ressentir ce qu'ils ressentent ?). Troisièmement, certains expriment leurs réactions en fonction de 
stratégies auto-représentationnelles et en fonction de leur interlocuteur («face à ma fille je voulais rester rassurant »). Dès les premières minutes, l'individu cherche à donner à la situation de réception de l'événement « une bonne forme » facilement mémorisable afin de pouvoir à court, moyen ou long terme, le récupérer plus facilement en mémoire et le narrer. Préparer cette narration concerne moins l'événement lui-même qu'une façon de parler de soi aux autres, de ce que la personne faisait à cette époque de sa vie. Le récit met en valeur une image de soi qui sera donnée aux autres.

Les individus cherchent également activement à co-construire des représentations cognitives via l'interaction sociale. La fonction socio-cognitive de cette communication permet, avant tout, de prendre réellement conscience de la réalité de la catastrophe et de l'ancrer dans le réel. C'est un processus interpersonnel de négociation de la référence ${ }^{16}$ par, notamment, un mécanisme de normalisation qui abouti à rendre ses propres représentations plus proches des normes du groupe d'appartenance.

Le troisième processus débute de sept à huit minutes après la première vision des images. C'est un fort sentiment de tristesse qui domine puis de peur, lorsque certains entendent les journalistes poser la question d'une éventuelle riposte américaine.

\section{La méthode d'Etude des Cognitions verbalisées concomitantes En Réception (ECER)}

La méthode ECER recueille et analyse les cognitions verbalisées concomitantes à la réception d'un dispositif de communication médiatique. On demande à une personne de regarder le message, seule, et «de dire à voix haute tout ce qu'elle pense et se dit dans sa tête ». On enregistre à la fois ses verbalisations «en direct » et le son du message médiatique de manière à pouvoir mettre en lien les verbalisations et les éléments du dispositif qui les ont déclenchés.

\section{Fondements théoriques et épistémologiques}

Nous avons conçu la méthode ECER en combinant les protocoles verbaux concurrents ${ }^{17}$ utilisés en ergonomie cognitive et la tâche de listage des pensées ${ }^{18}$ issue de la psychologie sociale. Son principe de base est que les verbalisations produites pendant la réception sont des observables du fonctionnement cognitif. Elles reflètent les «pensées » c'est-à-dire les traitements en mémoire de travail $^{19}$. Le caractère concomitant des verbalisations offre à la méthode ECER plusieurs avantages : - les «pensées » verbalisées étant plus spontanées, la méthode ECER limite les oublis (causés par

${ }^{16}$ R. GHIGLIONE, A, TROGNON, Où va la pragmatique ? - De la pragmatique à la psychologie sociale - Grenoble, Presses Universitaires de Grenoble, Vies Sociales, 1993

17 A.K. ERICSSON, H.A. SIMON, Protocol Analysis: Verbal reports as data (Rev. Ed.). London: MIT Press, 1993.

18 A.G. GREENWALD, « Cognitive learning, cognitive response to persuasion, and attitude change », in A.G. GREENWALD, T.C. BROCK, T.M. OSTROM (Eds.), Psychological foundations of attitudes, San Diego, CA, Academic Press, p. 147-170, 1968.

${ }^{19}$ H. KUUSELA, P. PAUL, Op. Cit. 
l'effacement en mémoire de travail), les rationalisations a posteriori et la centration artificielle des pensées sur le contenu argumentaire souvent observés dans la tâche de listage des pensées (Greenwald, 1968) qui a lieu après la réception ;

- la méthode ECER n'empêche pas mais facilite le traitement cognitif du dispositif car l'acte de «penser dans sa tête » et la verbalisation à voix haute sont gérés par le même code verbal. Après une phase d'entraînement, l'individu ne passe plus par les deux étapes "penser mentalement » puis «exprimer à haute voix la pensée » mais pense directement à haute voix ${ }^{20}$.

Souhaitant donner à la méthode ECER une bonne validité théorique quant au lien entre la cognition et le langage, nous n'analysons pas les verbalisations concurrentes avec l'analyse de contenu thématique, insuffisamment validée sur le plan théorique mais avec l'Analyse Cognitivo-Discursive (ACD), en partie automatisée par le logiciel Tropes. L'analyse des verbalisations, plus valide, gagne en précision, en rapidité et en objectivité.

La méthode ECER a un double fondement théorique : elle s'appuie à la fois sur un modèle du sujet communiquant et sur un modèle de réception expliquant son interaction avec le dispositif médiatique en situation de réception. Le récepteur, doté d'intentionnalité, met en œuvre une activité psychologique consciente et non consciente, plus ou moins élaborée mais toujours d'une grande complexité, où il traite en parallèle et en interaction des informations de nature cognitive et affective. Pour produire la signification qui contribuera à former, renforcer ou changer des comportements, il sélectionne les informations, comprend, infère des intentions informatives et persuasives, juge et réagit affectivement au contenu. Ces processus intra-individuels dépendent de caractéristiques individuelles et psychosociales (motivations, implication, attitudes et représentations préalables) et du contexte socio-spatio-temporel de réception.

Pour illustrer la méthode, nous l'appliquons à un contexte de communication persuasive où les méthodes de recherche figurant dans la littérature ne permettent pas de comprendre précisément le rôle joué par l'implication du récepteur sur les traitements du dispositif de communication. Deux modèles s'opposent. Selon le modèle ELM (Elaboration Likelihood Model $^{21}$ ), le récepteur impliqué traite de façon élaborée les arguments du message, appelés indices centraux (traitement central) et le récepteur peu impliqué traite de façon peu élaborée des indices dits périphériques, c'est-à-dire des éléments autres que les arguments du message. Le modèle à processus unique (unimodel ${ }^{22}$ ) considère que le récepteur peut, quel que soit son niveau d'implication, traiter de façon plus ou moins élaborée les deux types d'indices, centraux et périphériques. Il envisage donc un processus unique de persuasion qui ne différencie pas la force des traitements (niveau d'élaboration) et le contenu traité.

\footnotetext{
${ }^{20}$ B. RYAN, C.M. HASLEGRAVE, « Use of concurrent and retrospective verbal protocols to investigate workers' thoughts during a manual-handling task », Applied Ergonomics, 38-2, p.177-190, 2007

${ }^{21}$ R.E. PETTY, J.T. CACIOPPO, « The Elaboration Likelihood Model of Persuasion », in L. BERKOWITZ (Ed), Advances in Experimental social Psychology, Vol 19, San Diego, CA, Academic Press, p. 123-205, 1986.

${ }^{22}$ W.Y. CHUN, S. SPIEGEL, A.W. KRUGLANSKI, « Assimilative behavior identification can also be resource dependent: A Unimodel-Based Analysis of Dispositional Attribution Phases », Journal of Personality and Social Psychology, 83, p. 542$555,2002$.
} 


\section{L'implication dans la réception de la communication persuasive}

La méthode ECER est mobilisée pour départager le modèle ELM et le modèle à processus unique dans un contexte de communication politique. Elle vise à comprendre comment le degré d'implication d'un individu dans un thème ${ }^{23}$ affecte la réception d'un message. On compare les traitements issus des deux groupes de sujets ayant un niveau d'implication différent (la variable psychosociale est alors une variable indépendante -VI- à deux modalités). Nos hypothèses vont dans le sens du modèle à processus unique. Dans la première hypothèse, on s'attend à ce que les individus impliqués aient un traitement plus élaboré que les individus faiblement impliqués et donc qu'ils émettent davantage de cognitions verbalisées concomitantes. Dans la deuxième hypothèse, on s'attend à ce que plus les individus sont impliqués, plus ils émettent de cognitions verbalisées concomitantes, concernant à la fois le contenu argumentaire (indices centraux) et des indices périphériques. Dans la troisième hypothèse, on s'intéresse aux stratégies cognitivo-discursives ${ }^{24}$ marquant la relation du sujet au monde, mises en scène avec des marqueurs discursifs spécifiques ${ }^{25}$. On s'attend à ce que, dans leurs verbalisations, les sujets fortement impliqués vs. non impliqués mettent en scène un monde réel (type 1) vs. un monde possible (type 2).

\section{Procédure pratique de passation, de recueil et matériel}

La procédure expérimentale dure 30 minutes en moyenne et se déroule en plusieurs étapes.

On indique au sujet qu'il va voir la conférence de presse donnée par le "Président de la commission interministérielle sur les phénomènes de pollution atmosphérique urbaine qui mène une étude nationale sur les problèmes de circulation et de pollution en zone urbaine". Il y annonce des mesures de lutte contre la pollution. On fait deux groupes de sujets. Dans le groupe «forte implication» (première modalité de la variable indépendante), on indique que les mesures annoncées les concernent directement car elles seront mises en place autour de leur université 2 mois plus tard. Dans l'autre groupe «faible implication» (deuxième modalité de la variable indépendante), on indique que ces mesures ne les concernent pas car elles seront mises en place autour de leur université dans 7 ans.

On donne la consigne suivante: «Avant de montrer le message qui suit au grand public, nous souhaitons recueillir vos réactions, quelles qu'elles soient. C'est pour cela que je vous demande

\footnotetext{
${ }^{23}$ M.P. FOURQUET-COURBET, D. COURBET, « Nouvelle méthode d'étude des cognitions en réception (ECER) et application expérimentale à la communication politique », Revue Internationale de Psychologie Sociale, Tome 17, $\mathrm{n}^{\circ} 3$ (Septembre 2004), p.27-75, 2004.

${ }^{24}$ Dans la stratégie cognitivo-discursive de type 1, le but est lié à des réalités à affirmer ou, en d'autres termes à une clôture immédiate du monde possible projeté sur le monde réel. La stratégie 2 est liée à un but de réalités à construire et la clôture du monde possible projeté sur le monde réel est, dans ce cas, progressive. La stratégie 3 est associée à des réalités à comparer ou à justifier : elle traduirait l'ouverture d'un monde possible projeté sur d'autres mondes possibles.

${ }^{25}$ R. GHIGLIONE, A, TROGNON, Op. Cit.
} 
d'essayer de dire à voix haute ce que vous pensez, au moment où vous le pensez [...]».

On laisse le sujet seul dans la pièce. Le matériel est un document audiovisuel de $10 \mathrm{mn} 40 \mathrm{~s}$. Un acteur joue le rôle du Président de la commission. Il annonce des mesures de lutte contre la pollution et notamment des restrictions de circulation drastiques sur le campus universitaire de la ville où a lieu l'expérience : tous ses arguments sont des indices centraux. Un certain nombre d'indices périphériques susceptibles d'attirer l'attention et d'être traités ont été volontairement intégrés au dispositif de communication médiatique (e.g. mimiques faciales, lapsus de l'acteur, sonneries de téléphone pendant le discours, erreurs de cadrage) ${ }^{26}$.

Enfin au cours d'un débriefing, on annonce les objectifs réels de l'étude au sujet et on s'assure de la crédibilité de la situation et du matériel. On demande également, à une sélection de sujets de l'échantillon choisis aléatoirement, de donner leurs impressions sur la tâche de penser à voix haute.

\section{Echantillon}

103 étudiants de l'université de Nice-Sophia-Antipolis ont été recrutés pour participer à l'expérience (77 femmes et 26 hommes). Ils ont été aléatoirement répartis dans les deux groupes expérimentaux (53 en condition de forte implication et 50 en condition de faible implication).

\section{Retranscription et méthode d'analyse des discours}

Le chercheur analyse les discours sans connaître le groupe auquel appartient le sujet. Après retranscription, il faut identifier les déclencheurs (indices centraux ou périphériques) et la valence (favorable / neutre / défavorable au dispositif) de chaque réponse cognitive. Le discours est ensuite analysé avec le logiciel Tropes afin d'identifier :

- les catégories discursives utilisées (verbes, adjectifs, modalisations, joncteurs,...) qui permettent de détecter les stratégies cognitivo-discursives des sujets et leurs rapports au monde, la prise en charge du discours par le sujet et sa subjectivité ;

- les références et les univers de référence.

\section{L'implication produit des discours plus riches}

Le tableau 2 reprend les résultats les plus significatifs. Par rapport aux non impliqués, les sujets impliqués ont déclenché significativement plus de réponses cognitives, réagi davantage aux indices centraux et aux indices périphériques et utilisé davantage catégories discursives telles que les verbes statifs, les modalisations d'intensité et le mode indicatif.

\footnotetext{
${ }^{26}$ Un pré-test nous a assuré de la crédibilité du message malgré l'extrémisation des indices.
} 
Tableau 2 : Principaux effets de l'implication sur les traitements du dispositif médiatique

\begin{tabular}{|c|c|c|c|c|}
\hline Variables & $\begin{array}{c}\text { Groupe } 1 \\
\text { impliqués } \\
\text { Moyenne } \\
\text { (écart-type) }\end{array}$ & $\begin{array}{c}\text { Groupe } 2 \\
\text { non impliqués } \\
\text { Moyenne } \\
\text { (écart-type) }\end{array}$ & t de Student & $\begin{array}{c}\text { Signification } \\
P^{*}\end{array}$ \\
\hline \multirow{2}{*}{\multicolumn{5}{|c|}{$\begin{array}{c}\text { NIVEAU } \\
\text { D'ELABORATION }\end{array}$}} \\
\hline & & & & \\
\hline Nombre d'interventions & $21,9(12,50)$ & $16,7(11,54)$ & $-2,17$ & .03 \\
\hline Nombre de réponses cognitives & $20,1(12,14)$ & $14,9(10,81)$ & $-2,27$ & .02 \\
\hline \multicolumn{5}{|l|}{ DECLENCHEURS } \\
\hline Nombre de déclencheurs=indices périphériques & $18,9(10,86)$ & $14,7(10,13)$ & $-2,02$ & .04 \\
\hline Nombre de déclencheurs=indices centraux & $50,7(25,10)$ & $40,1(23,38)$ & $-2,20$ & .03 \\
\hline \multicolumn{5}{|l|}{ CATEGORIES DISCURSIVES UTILISEES } \\
\hline Verbes statifs & $0,08(0,02)$ & $0,07(0,03)$ & 3,18 & .001 \\
\hline Modalisations d'intensité & $0,04(0,02)$ & $0,03(0,02)$ & 2,23 & .02 \\
\hline Mode indicatif & $0,125(0,02)$ & $0,113(0,04)$ & 1,92 & .05 \\
\hline Pronom " nous" & $0,003(0,04)$ & $0,001(0,002)$ & 2,53 & .01 \\
\hline Pronom "vous" & $0,001(0,0004)$ & $0,001(0,003)$ & $-2,29$ & .02 \\
\hline Pronom “"on” & $0,02(0,01)$ & $0,01(0,09)$ & 2,16 & .03 \\
\hline \multicolumn{5}{|l|}{ STRATEGIES COGNITIVO-DISCURSIVES } \\
\hline Type 1 : " réalité à affirmer" & $0,4(0,05)$ & $0,3(0,09)$ & 2,9 & .004 \\
\hline Type 2 : " réalité à construire" & $0,4(0,05)$ & $0,3(0,10)$ & 1,93 & .05 \\
\hline
\end{tabular}

Note. * l'hypothèse est confirmée quand p est inférieur à $.05:$ le seuil de risque est alors à $5 \%$.

L'implication agit sur le niveau d'élaboration du traitement cognitif du dispositif médiatique mais non sur sa nature : les sujets impliqués ont émis un nombre plus important de réponses cognitives pendant la réception. Ils ont davantage traité aussi bien les arguments (indices centraux) que les éléments périphériques. Les résultats confirment nos hypothèses qui postulent l'existence d'un seul processus de la persuasion intégrant à la fois et indépendamment la force et la nature du traitement. Dans le cadre de cette étude, ils montreraient donc la supériorité du modèle à processus unique sur le modèle ELM. L'implication semble également favoriser l'expression d'une stratégie cognitivo-discursive de type 1, c'est-à-dire d'une « réalité à affirmer » où le sujet exprime un «je sais que » et procède donc à une clôture immédiate du monde possible projeté sur le monde réel. 


\section{Analyse comparative des apports des 2 méthodes RRR et ECER}

L'analyse comparative des deux méthodes discute leur intérêt épistémologique et théorique et établit les conditions nécessaires pour garantir leur validité et leur bonne application, dans le cadre des études de la réception de la communication médiatique.

\section{Intérêts et apports épistémologiques}

A un niveau général, les deux méthodes se fondent sur une même théorie complexe des phénomènes communicationnels. Mais leur manière de les étudier ces phénomènes diffèrent. L'approche compréhensive sous-tend la méthode des RRR. Elle postule que l'intervieweur a la possibilité de pénétrer le vécu et le ressenti d'une autre personne. Elle implique des moments de saisie intuitive, par empathie avec les significations et les affects des faits de réception médiatiques, en tant que phénomènes humains et sociaux. Le RRR peut soit :

- explorer le phénomène de manière empirico-inductive;

- évaluer différentes explications ou hypothèses alternatives sur la réception, éventuellement déduites de théories ;

- expliciter des hypothèses qui seront plus tard testées sur d'autres phénomènes et par d'autres méthodes.

Avec la méthode ECER, le chercheur a deux stratégies de recherche possibles. Premièrement, il peut l'intégrer dans une démarche herméneutique pour comprendre comment les sujets sociaux construisent la signification en interaction avec un dispositif médiatique. Deuxièmement, il peut tester des modèles explicatifs et causaux : la méthode est alors intégrée à un plan expérimental pour tester le rôle et les effets de variables pertinentes. La démarche permet alors de construire des modèles à forte valeur explicative où seront recherchées les déterminants des traitements socio-affectifs et socio-cognitifs (et non plus la signification subjective), en suivant la double logique épistémologique de confirmabilité et de la falsification d'hypothèses ${ }^{27}$. La méthode des RRR, quant à elle, ne peut falsifier des hypothèses, au sens fort, mais uniquement confirmer des hypothèses en cumulant les observations.

\section{Les principes théoriques et les principes opérationnels}

Pour les deux méthodes, il faut distinguer les principes théoriques des principes opérationnels de base. Dans la méthode RRR, le chercheur adhère au principe théorique selon lequel la réception est coconstruite par et dans un récit. Le souvenir récupéré en mémoire dépend par exemple de l'humeur de l'interviewé au moment de la récupération et du cadre psychosocial de l'enquête, notamment de l'interaction avec l'intervieweur. D'un point de vue opérationnel et s'il veut mener sa recherche empirique, le chercheur accepte, peu ou prou, le principe selon lequel le RRR est un quasi reflet de la réalité. Les recherches sur la mémoire flash montrent qu'au fil du temps et malgré les multiples

\footnotetext{
${ }^{27}$ K. R. POPPER, La logique de la découverte scientifique, Traduction française, 1973, Payot, 1935
} 
narrations faites par le sujet, les récits se modifient très peu ${ }^{28}$ Pour la méthode ECER, sur un plan théorique, le principe de co-construction du discours vaut également : la méthode ne peut enregistrer « fidèlement » les pensées du récepteur, à cause notamment du contexte et des attentes expérimentales. D'un point de vue opérationnel, on accepte le principe selon lequel les verbalisations du sujet social reflètent les activités cognitives en cours.

\section{Champs d'application et procédures pour s'assurer d'une bonne recherche avant le recueil}

\section{Champs d'application}

Si le champ d'application de la méthode RRR est réduit aux événements socio-médiatiques, historiquement uniques, saillants, impliquants pour les individus et suscitant de fortes émotions, le champ d'application de la méthode ECER est plus large. Celle-ci permet l'étude de la réception de divers types de dispositifs médiatiques réels (avec d'éventuelles applications industrielles comme les études qualitatives de programmes ou de publicités télévisuels) ou construits pour les besoins d'une recherche.

\section{Contraintes pratiques de passation}

On ne dispose pas de suffisamment de recherches pour connaître le délai « maximal convenable » entre l'événement médiatique et l'enregistrement du RRR. Si un délai trop court fait courir le risque d'avoir des récits trop «chauds », c'est-à-dire sans prise de recul nécessaire à une mise à distance raisonnée du phénomène de réception, un délai trop long risque de biaiser le souvenir. Deux mois semblent un délai «optimal convenable » pour les événements saillants ${ }^{29}$. Si la méthode ECER est pratique et facile à mettre en place (pas de contraintes de délai ou liées au lieu de passation), la méthode RRR est plus coûteuse matériellement car les entretiens doivent se passer sur le lieu de la réception, ce qui nécessite le déplacement des enquêteurs à domicile.

\section{Echantillon}

La méthode des RRR nécessite un échantillon diversifié mais non représentatif : 20 à 30 personnes interrogées donnent 90 à 100\% des informations ${ }^{30}$. Dans l'enquête sur les attentats de New York, le point de saturation a été atteint à 30 sujets : à partir du $30^{\text {ème }}$ RRR, l'analyse de RRR supplémentaires n'a pas occasionné une meilleure compréhension du phénomène. Pour la méthode ECER, l'automatisation partielle par logiciel de l'analyse des discours permet l'étude d'un échantillon élevé de sujets choisis selon les objectifs de l'étude. Dans le cas d'un plan expérimental, deux

\footnotetext{
${ }^{28}$ D.B. WRIGHT, G.D. GASKELL, op.cit.

${ }^{29}$ H.T. REIS, S.L. GABLE, « Event Sampling and other methods for studying daily experience », in H.T. REIS, C. JUDD

(Eds.), Handbook of research methods in social and personality psychology, NY Cambridge University Press, p. 190-222, 2000.

30 A. GRIFFIN, J.R. HAUSER, « The Voice of The Customer », Marketing Science, vol.12, N 1 , p.1-27, 1993.
} 
caractéristiques de l'échantillon garantissent une bonne validité interne : une taille suffisante pour que les tests statistiques soient valables (environ 30 sujets minimum par groupe, permettant d'avoir des seuils de risque inférieur à 5\%) et une affectation aléatoire des sujets dans les groupes expérimentaux afin de contrôler les variables non pertinentes.

\section{Comment s'assurer d'une bonne recherche pendant le recueil des données ?}

Lors des RRR, au cours de l'interaction avec le récepteur, le savoir-faire de l'enquêteur est primordial pour saisir en compréhension la «psychologie » de l'interviewé et le contexte de l'entretien lui-même (ce qui permet de maximiser la «validité de portrait »). L'objectif est de maximiser, premièrement, la validité d'histoire, permettant d'affirmer que le récit fait par les répondants est complet, vrai, authentique et sincère ${ }^{31}$. C'est par la qualité de la relation que l'intervieweur établit avec l'interviewé (climat de confiance, bonne gestion des interactions, qualité de l'écoute et des relances, capacité à adapter le guide d'entretien...) que l'on va chercher à accrô̂tre, deuxièmement, la «validité expérientielle» des RRR. Il s'agit d'obtenir des données très riches afin d'arriver à la complétude, c'est-à-dire à enregistrer l'expérience émotionnelle et le vécu des individus dans toutes leurs dimensions, pendant la réception mais également après l'événement, de manière à évaluer de manière valide (sans le sur ou sous-estimer) l'importance de l'événement, plus tard, dans la vie de la personne. Pour accroître la validité des RRR, plusieurs techniques peuvent être associées : le recoupement avec des sources télévisuelles (telle image a-t-elle bien été montrée au moment où l'interviewé nous le dit ?), la technique de la confirmation externe (on demande à d'autres personnes impliquées dans le récit du premier individu interviewé leur version de la situation) ou la preuve par la triangulation des sources. En cas d'absence de concordance, la souplesse de la méthode permet de faire appel, $a$ posteriori et de manière $a d$ hoc, au principe de la «cristallisation » par lequel on accepte qu'un même phénomène puisse être expliqué différemment par des personnes différentes ${ }^{32}$.

Si la méthode ECER est intégrée à un plan expérimental, le comportement de l'interviewer doit rester le plus standardisé possible afin que la seule différence entre les groupes expérimentaux provienne des variables indépendantes que l'on manipule. Pour éviter les biais de valorisation sociale, il est préférable que le sujet soit seul lors de la réception. Le matériel d'enregistrement doit être discret. L'expérimentateur doit s'assurer que la consigne de «penser à voix haute » a bien été comprise («je vous demande simplement de dire à voix haute ce que vous vous diriez habituellement dans votre tête, n'en rajoutez pas, ne vous censurez pas »). Une phase d'entraînement à partir d'un message quelconque permet par ailleurs d'accroitre la validité interne des protocoles concurrents ${ }^{33}$.

\footnotetext{
31 J.F. SHERRY, J.W. SHOUTEN, « A role for poetry in consumer research », Journal of Consumer Research, 29, 2, p. 218234, 2002

32 L. RICHARDSON, «Writting: A Model of Inquiry », In N.K. DENZIN, Y.S. LINCOLN et Al., Handbook of Qualitative Research, Sage, $2^{\text {nd }}$ Ed., 2000.

${ }^{33}$ RYAN, 2007, Op. Cit.
} 


\section{Comment s'assurer d'une bonne analyse des données et d'une bonne validité des résultats après}

\section{le recueil ?}

\section{Validité interne, validité d'interprétation}

Dans l'analyse des données des RRR, deux écoles s'opposent sur le caractère subjectif des analyses. L' « école subjectiviste » recommande que ce soit l'intervieweur qui fasse lui-même l'analyse afin d'y intégrer son vécu subjectif et les impressions qu'il a eues au cours de l'entretien. La validité d'interprétation des RRR, difficilement objectivable, dépend alors de la capacité de l'analyste à comprendre les sujets interrogés, leur vécu en contexte et leur «psychologie profonde». L'interprétation subjective sera d'autant plus riche que le chercheur aura lui-même vécu l'événement médiatique dans les mêmes conditions que les sujets interviewés. On demandera à l'analyste que l'interprétation des résultats ait tout de même une bonne cohérence interne, c'est-à-dire qu'elle ne se contredise pas.

Pour l'école « objectiviste », la subjectivité des analystes des RRR doit être réduite au minimum. Pour cela, on sépare la description de linterprétation des résultats. Cette pratique offre notamment deux avantages. Elle permet de réanalyser, plus tard, les résultats à la lumière d'un autre contexte théorique pouvant éventuellement mettre en évidence d'autres types d'interprétations théoriques. Elle permet, en outre, d'augmenter le nombre de codeurs pour accroître l'objectivité des analyses. Le calcul d'un coefficient de fidélité intercodeurs contribue à contrôler la «qualité » de l'analyse de contenu et à l'améliorer au besoin.

Pour accroître la validité d'interprétation des RRR, on valide deux critères. Pour valider le critère d'acceptation interne, après avoir retranscrit et analysé le RRR, on demande au sujet son degré d'accord avec l'interprétation de son récit. Pour valider le critère de cohérence interne, on demande à une personne extérieure à la recherche si la logique de progression dans l'analyse et l'interprétation lui semble explicite et rationnelle.

La validité interne de la méthode ECER concerne sa valeur relativement à son propre cadre et à ses propres objectifs. La méthode expérimentale permet d'atteindre un haut degré de validité interne en limitant les effets de variables parasites contaminant la mise à l'épreuve des hypothèses. Pour cela, en plus d'une randomisation de l'affectation des sujets dans les groupes, il faut mettre en place une validation en double-aveugle. Premièrement, les sujets ne sont pas tenus au courant de la façon dont les variables indépendantes sont manipulées. Aussi, le comportement de l'expérimentateur doit-il être identique dans tous les groupes. Deuxièmement, lorsqu'il analyse les données, le codeur ne doit pas savoir à quel groupe expérimental appartiennent les sujets.

\section{Généralisation et validité externe}

Pour savoir si l'on peut généraliser les résultats, les critères diffèrent pour les RRR et pour la méthode ECER. Pour les RRR, c'est la validité de généralisation qu'il convient de viser. Celle-ci est bonne 
lorsque le chercheur parvient à donner du sens à l'ensemble du corpus en en dégageant, sans en réduire la richesse, une structure invariante qui se répète en dehors des cas particuliers. Elle est souvent convenable si le chercheur est parvenu à atteindre le point de saturation, ce qui confère à l'étude une bonne transférabilité. C'est le cas si la récolte des données a été faite auprès d'un échantillon de récepteurs diversifiés et que le contexte local de réception a été décrit de façon détaillée. Plus précisément, derrière la généralisation, on recherche une bonne «transférabilité des résultats » ${ }^{34}$, c'està-dire la possibilité de transférer les résultats à d'autres circonstances et à d'autres événements médiatiques. L'évaluation de la transférabilité est le fruit d'une discussion entre plusieurs experts où chacun donne son avis en fonction de son expérience propre et de la connaissance qu'il a du nouveau contexte. S'il y a accord entre experts, la transférabilité des résultats est obtenue par un processus de validation communicationnelle ${ }^{35}$.

Quand la méthode ECER est intégrée à un plan expérimental, la généralisation des résultats est bonne quand la validité externe est bonne. Cette dernière est composée de la validité échantillonnale et de la validité écologique. On essaye alors de savoir dans quelle mesure et pour quelles raisons empiriquement étayées, les résultats obtenus peuvent-ils (ou ne peuvent-ils pas) être étendus à d'autres types de population (validité échantillonnale) ou à d'autres types de contexte de réception (validité écologique).

\section{Conclusion}

Enrichissant l'arsenal méthodologique des sciences de la communication, ces deux méthodes devraient contribuer à mieux connaître les processus de réception lors du contact avec une large variété de dispositifs et messages médiatiques. La méthodologie est d'une importance épistémologique cruciale pour les sciences de la communication dans la mesure où elle assure à la fois la double transition du terrain aux théories et la validité des connaissances construites.

Parmi les nouvelles perspectives de recherche, deux nous semblent prioritaires. Une grande partie des processus de réception ne sont pas conscients mais sont mis en route automatiquement par le système cognitif quand les personnes sont face aux médias. Il conviendrait d'étudier le rôle des automatismes cognitifs et affectifs (formation de stéréotypes et de cognition implicites, conditionnement évaluatif, modelage automatique ${ }^{36}$ ) dans la production des verbalisations concomitantes ou rétrospectives. Le rôle de la rationalisation

\footnotetext{
${ }^{34}$ A. GUBA, « Criteria for assesing the truthworthiness of Naturalistic Inquiry », Education, Communication and Technology, vol.29, p.75-91, 1981

35 N.K. DENZIN, Y.S. LINCOLN et Al., Handbook of Qualitative Research, Sage, 2nd Ed, 2000.

${ }^{36}$ BEAUVOIS, J.-L. , Les illusions libérales, individualisme et pouvoir social, Presses Universitaires de Grenoble, 2006.
} 
grâce à laquelle l'individu justifie a posteriori ses réactions, catégorisations et jugements générés automatiquement et sans conscience par son système cognitif est aussi à mieux connaître. De telles perspectives nécessiteraient l'utilisation de la méthode expérimentale. Ainsi, le caractère pluridisciplinaire des sciences de la communication ne conduit pas uniquement à mobiliser différentes ressources théoriques, mais aussi à croiser des méthodes différentes, qu'elles soient qualitatives, quantitatives ou expérimentales. Afin d'accroître de manière heuristique le nombre d'objets étudiés et la validité des connaissances scientifiques construites, le développement des recherches sur les médias passe par la défense d'un pluralisme théorique, méthodologique et épistémologique. 